\title{
NÉCTAR DE TUMBO (Passiflora mollisima HBK)
}

Enrique De Florio Ramírez

\author{
RES U M E N
}

Se ha estudiado al frutal nativo, tumbo serrano (Passiffora mollisima), para elaborar néctar:

La composición promedio y estudio biométrico del mismo son reportados en la parte bibliográfica.

Se deterninaron parámetros para su elaboración: indice de madurez del tumbo 7,21. Cortado longitudinal. Dilución 5 partes de agua por parte de jugo, llevaindola a I5 Bra: Llenado al vacio. Pasteurizado a $85^{\circ} \mathrm{C}$ por espacio de 15 minutos. Se reporta la caracterización del néctar de tumbo.

\section{A B S T R A C T}

The native fruit tree, tumbo serrano (from the highlands), has been studied for nectar preparation.

The rate composition and biometrics study is reported in the bibliographical part.

The following parameters were determined for its preparation: 7,21 ripeness rate, manual selection, longitudinal cut, diluted and pasteurized at $85^{\circ} \mathrm{C}$ for $15 \mathrm{~min}$. The tumbo nectar's characterization is reported.

\section{INTRODUCCIÓN}

El tumbo(Passiflora mollisima H.B.K. Bailey) es un frutal nativo que tiene una producción agrícola pequeña y que se desarrolla en zonas andinas, entre los 2000 a 3500 msnm. También se le cultiva en Nueva Zelanda y Hawai. (Calzada, 1980).

El objetivo general del presente trabajo es el de favorecer al desarrollo de la agroindustria del frutal nativo tumbo en la subregión Tacna. son:

Los objetivos especificos del presente trabajo

- Caracterizar al tumbo como materia prima para néctares.

- Determinación de parámetros óptimos para la elaboración de néctar de tumbo

1. Ing. en Industrias Alimentarias

\section{REVISIÓN BIBLIOGRÁFICA}

\subsection{ASPECTOS GENERALES DEL TUMBO}

\subsubsection{Clasificación botánica}

Passiflora mollisisima H.B.K. Bailey.

\subsubsection{Sinonimias}

Tumbo serrano y tacso (Perú), curuba (Colombia), parcha (Venezuela) granadilla cimarrona (México), taxo (Ecuador). Internacionalmente se le conoce también con el nombre de banana passion fruit, soltleaf passion flower (Calzada, 1980).

\subsubsection{Composición Química}

El tumbo es muy rico en niacina, caróteno $1.2 \mathrm{mg}$, y vitamina $\mathrm{C}$, como apreciamos en el Cuadro $\mathrm{N}^{\circ} 1$.

\subsubsection{Usos}

Esta fruta se vende solamente en estado 
fresco, pero Calzada (1980) la recomienda para la elaboración de mermeladas y preparación de aperitivos.

\subsubsection{Producción}

La producción está limitada a la superficie de 2 hectáreas, con un rendimiento por hectárea de $4000 \mathrm{~kg}$. Dando una producción de $8 \mathrm{tm}$ para todo el departamento de Tacna. (Anuario Estadístico del Perú,1986).

CUADRO No 1: Composición química del tumbo serrano.

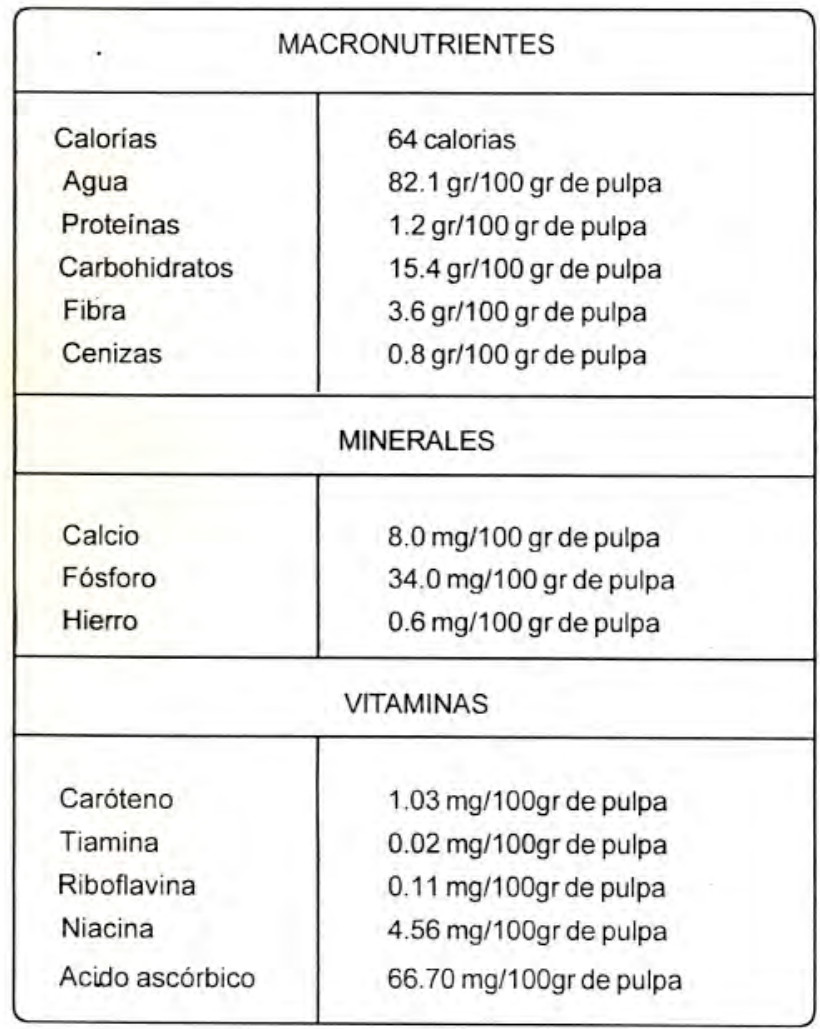

Fuente:Collazos et al (1986)

\subsubsection{Caracteristicas de la materia prima}

De Florio (1995) realizó mediciones biómetricas en el fruto y jugo del tumbo (Passiflora mollisisima H.B.K. Bailey), procedente del anexo de Ticaco, provincia de Tarata, obteniéndose los siguientes resultados:

a) Materia prima.- La materia prima fue obtenida en el mercado de productores de Tacna, procedente del anexo de Ticaco, provincia de Tarata, la misma que se lavó y evaluó, obteneniéndose los siguientes resultados:
- Apariencia externa:

Color

Amarillo pálido

Olor

Aromático característico

- Medidas biométricas:

Forma

Elíptica

Longitud

$7.86 \mathrm{~cm}$

Ancho

$3.38 \mathrm{~cm}$

Peso promedio

$70.46 \mathrm{gr}$

- Rendimientos:

Pulpa $388.4987 \mathrm{gr}$

Cáscara $161.02 \mathrm{gr}$

Pepas y fibra $380.71 \mathrm{gr}$

Rendimiento total: $41.76 \%$

- Características de la pulpa:

Organolépticas:

$\begin{array}{ll}\text { Color } & : \text { Naranja ,opaco } \\ \text { Olor } & : \text { Muy aromático } \\ \text { Sabor } & : \text { Agradable, muy ácido, sui } \\ & \text { generis, algo astringente. }\end{array}$

Físico-Químico es:

$\mathrm{pH}$

Sólidos solubles

: $3.55-3.78\left(25^{\circ} \mathrm{C}\right)$

Acidez titulable : $1.65 \%$ (como ácido cítrico)

Densidad relativa : 1.04

Indice de madurez : 7.29

Pectina

: Presencia de pequeña cantidad (medida cualitativamente).

La madurez óptima del tumbo se encuentra con un índice de 7.29.

\subsection{ELABORACIÓN DE NÉCTARES DE FRUTAS}

Muchas frutas son demasiado ácidas o de sabor muy fuerte para ser agradables al beberse sin diluir. A menudo, estos fuertes jugos agridulces son deliciosos después de una dilución con un jarabe o jugo soso. Ejemplo de estos jugos de este tipo son: arándalo, frambuesas y la mayoria de estos jugos de bayas, ciruelas y guayaba.

Se define néctar (ITINTEC,1984) como constituido por jugo de fruta finamente dividido y tamizado, diluido con agua con la adición de edulcorante y si es necesario un ácido orgánico apropiado, convenientemente elaborado y sometido a un tratamiento térmico que asegure la conservación en un envase hermético. 


\section{MATERIALES Y MÉTODOS}

El presente trabajo se realizó en los laboratorios de la Facultad de Industrias Alimentarias de la Universidad Nacional Jorge Basadre Grohmann de Tacna.

La materia prima que se utilizó, tumbo (Passiflora mollisima H.B.K.) procedente del anexo de Ticaco, provincia de Tarata subregión Tacna.

\subsection{MATERIALES}

Reactivos químicos y medios de cultivo microbiológico

Material de vidrio

Estufas

Potenciómetro

Refractómetro manual

Cocina

Agua San Luis, etc.

\subsection{MÉTODOS}

\subsubsection{Diseño Experimental}

El diagrama de flujo tentativo se encuentra en la Fig. $N^{\circ} 1$, con la que se evaluó los parámetros en el presente trabajo de investigación, el que a continuación se detalla:

- Materia prima: Se evaluará el grado de madurez $\mathrm{pH}$.

- Lavado: Manual

- Cortado: Se aplicará un corte longitudinal para facilitar la extracción.

- Extracción: El contenido del fruto será extraido en forma manual, con la ayuda de una cuchara y utilizando un tamiz se presionará las papilas de la pulpa contra las paredes, para separarlas de las semillas, teniendo cuidado de no fraccionar las últimas para evitar la aparición de una astringencia marcada e impurezas (puntos negros), como se encontró en experimentos preliminares cuando se utilizó la licuadora a bajas velocidades.

- Estandarización o normalización del néctar: Entre los ajustes previstos a relizarse para el néctar, se encuentran:

a) Dilusión del jugo con agua tratada para bebida. Se determinará la dilución óptima entre la siguiente composición de muestras: 3:1 (tres
FIGURA $N^{\circ}$ 1: Flujo tentativo para la elaboración de néctar de tumbo

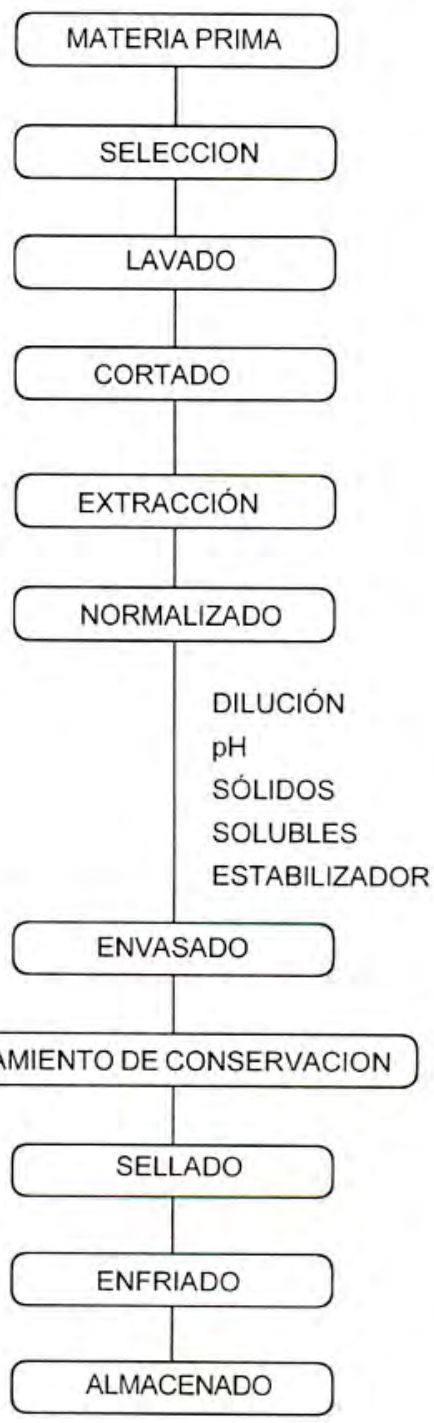

Fuente: Elaboración propia

partes de agua por una parte de jugo de tumbo), 4:1, 5:1, 6:1, 7:1.

b) Adición de edulcorante. A estas muestras se les llevará a una composición de sólidos solubles de $15^{\circ}$ Brix, mediante un balance de materia y la adición de sacarosa comercial.

c) $\mathrm{pH}$. Este valor se corregirá en caso de ser mayor a 4,0 con ácido citrico.

Una vez normalizado el néctar se aplicará el diseño estadistico de bloques completos al azar (Calzada 1966), para la determinación de la dilución óptima en base a la escala de 
hedónica de siete puntos y mediante la prueba de Duncan hallar la mejor muestra.

- Llenado: Se envasará la muestra con la dilución de jugo considerada como el mejor sabor en matraces de $125 \mathrm{cc}$ y en forma manual.

- Tratamiento de conservación: Se someterá a las muestras a tratamiento térmico, $85^{\circ} \mathrm{C} \times 15$ minutos; tratamiento químico: Benzoato de sodio $0.01 \% \mathrm{p} / \mathrm{v}+$ sorbato de sodio $0.021 \% \mathrm{p} / \mathrm{v}$. Tratamiento mixto : Tratamiento térmico $85^{\circ} \mathrm{C} \times 15$ minutos + benzoato de sodio $0.01 \%$, cuya composición se muestra en el Cuadro $\mathrm{N}^{\circ} 06$. En el mismo cuadro, se puede observar que los diferentes tratamientos serán evaluados mediante el análisis microbiológico para determinar su eficiencia.

- Sellado: Las muestras se sellarán herméticamente, una vez aplicado su tratamiento de conservación, utilizando tapones de jebe estériles.

- Enfriado: Se realizará en aquellas muestras que se les ha sometido a tratamiento térmico.

- Almacenamiento: Se realizará análisis químico - físicos a la muestra de néctar de tumbo, considerada como la óptima.

Con el flujo de elaboración definitivo se preparará muestras, a las cuales se les adicionará el estabilizador $\mathrm{CMC}$ en una cantidad igual a $0.06 \%$ y $0.01 \%$ y otra $\sin$ adicion de CMC (testigo), las cuales fueron llevadas a almacenamiento a temperatura ambiente y otra a $6^{\circ} \mathrm{C}$ con el objeto de evaluar la acción del estabilizado por espacio de 10 dias.

Se utilizará la altura de sedimento como factor discriminante

\subsubsection{Análisis Físico-Químicos}

A la muestra final de néctar de tumbo se realizará los análisis recomendados por Lees (1989):

- Acidez expresada en ácido cítrico

- Azúcares reductores

- Sólidos totales

$-\mathrm{pH}$

- Examen organoléptico

\subsubsection{Métodos microbiológicos}

Recuento total de gérmenes viables $(100 \mathrm{ml}$ y dilución).

Recuento de hongos y levaduras.

\subsubsection{Análisis organoléptico}

Diseño por bloques completos al azar (Calzada, 1966), siendo panelistas, alumnos del quinto año de la Facultad de Industrias Alimentarias de la Universidad Nacional Jorge Basadre Grohmann de Tacna, Promoción 1993.

\section{RESULTADOSYDISCUSIÓN}

\subsection{RENDIMIENTO DEL TUMBO}

El tumbo tuvo un rendimiento en pulpa de $40 \%$ y un indice de madurez de 7.21 .

\subsection{ENSAYO DEL ESTANDARIZADO.}

De la evaluación del ANVA se rechaza la hipótesis planteada y podemos concluir que en promedio no existe una diferencia significativa, en cuanto al sabor entre las diferentes diluciones aun nivel de á $=0.05 \%$.

Se aplicó la prueba de Tukey a un nivel del $5 \%$, encontrándose el mejor promedio en el tratamiento en la dilución, utilizando 5 partes de agua por una parte de pulpa o jugo 5:1.

La cantidad de sólidos solubles fue de $15^{\circ}$ Brix en todos los casos.

\subsection{TRATAMIENTO DE CONSERVACIÓN}

Los resultados del tratamiento de Conservación se observan en el Cuadro $\mathrm{N}^{\circ} 2$ y los que dieron mejor resultado son los que se utilizó

CUADRO N$^{\circ} 2$ : Evaluación de tratamiento de conservación.

\begin{tabular}{|l|c|c|}
\hline \multicolumn{1}{|c|}{ Muestra(Néctar de Tumbo) } & ANALISIS MICROBIOLOGICOS \\
\cline { 2 - 3 } & $\begin{array}{l}\text { RECUENTO } \\
\text { HONGOS } \\
\text { LEVADURAS }\end{array}$ & $\begin{array}{c}\text { RECUENTO } \\
\text { AEROBIAS } \\
\text { MESOFILAS }\end{array}$ \\
\hline $\begin{array}{l}\text { Tratamiento térmico } \\
85^{\circ} \mathrm{C} \times 15 \text { minutos }\end{array}$ & Ausente & Ausente \\
$\begin{array}{l}\text { Tratamiento térmico } \\
85^{\circ} \mathrm{C} \times 15 \text { min }+0.01 \\
\text { Benzoato de Sodio } \\
\text { Benzoato de sodio }(0.01 \%)+ \\
\text { Sorbato de potasio }(0.025)\end{array}$ & Ausente & Ausente \\
\hline
\end{tabular}


en tratamientos térmicos y de éstos, el mejor es el que no se adicionan conservantes quimicos.

\subsection{EVALUACIÓN DEL ESTABILIZADOR EN EL NÉCTAR DE TUMBO}

Los valores obtenidos de las observaciones diarias mantienen una cierta irregularidad para cada muestra y esto se debe a que el sedimento no es una capa homógenea, sino que tiene la tendencia a formar grumos, lo que dificulta la toma de medidas.

Se encontró que a los dos meses de conservaciòn, tanto a temperatura ambiente como en refrigeraciòn, la altura promedio del sedimento de todas las muestras fue de $6.5 \mathrm{~cm}$ y los valores estuvieron entre 0.5 y $0.8 \mathrm{~cm}$ por el método de extracción del néctar, y la falta de homogeneización.

También se observó que en las muestras almacenadas en refrigeración, la diferencia de altura de sedimento entre las muestras, no contiene CMC y las que si la contienen, es mayor que en el almacenamiento a temperatura ambiente.

La muestras sin CMC tiene menor altura de sedimento y las que la contienen tiene mayor altura que las similares almacenadas a temperaturas ambiente, debido a que en frío se precipitan más rápidamente los musilagos, pero se incrementa la viscosidad del estabilizador

\subsection{FLUJO DEFINITIVO}

En la Fig. $N^{\circ} 2$ se da el flujo definitivo y los parámetros óptimos para la obtención del néctar de tumbo

\subsection{CARACTERIZACIÓN DEL NÉCTAR DE TUMBO}

El néctar de tumbo se caracterizó obteniéndose el Cuadro $\mathrm{N}^{\circ} 3$, encontrándose que tiene un $\mathrm{pH}$ adecuado para su conservación por métodos térmicos de pasteurización.

En el mismo Cuadro $N^{\circ} 3$ se observa que la composición de su sólidos esta dado fundementalmente por azúcares sólubles.

\section{CONCLUSIONES}

1) El tumbo reúne las condiciones para ser procesado como néctar.

2) La extracción merece un gran cuidado por la posibilidad de romper semillas, cuyos componentes astringentes alteran el sabor del néctar de tumbo.

3) El rendimiento de fruto en jugo, mediante este método fue de $40 \%$.

4) Los parámetros del ajuste o normalizado del néctar son: Brix $15^{\circ}$, Dilusión de 5 partes de agua por una de jugo, ph 3.8-4.0

FIGURA $N^{\circ}$ 2: Flujo definitivo de operaciones para la elaboración de néctar de tumbo.

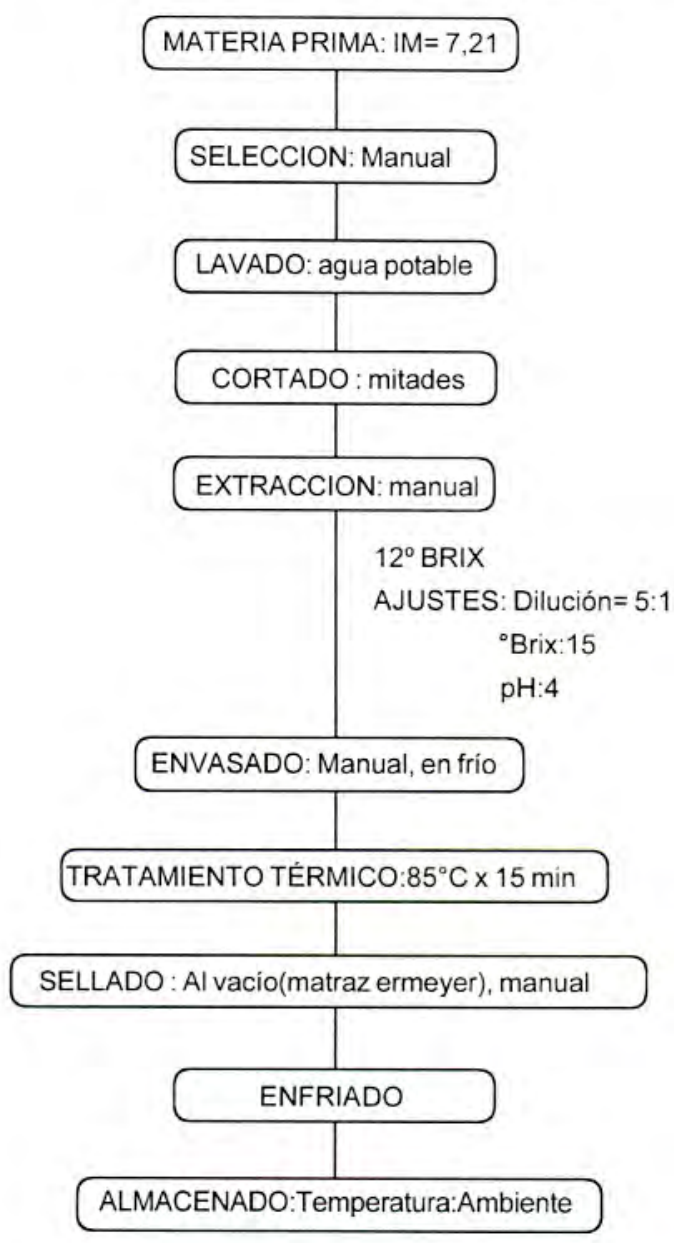

CUADRO N ${ }^{\circ}$ 3: Resultados de la caracterización del néctar de tumbo

\begin{tabular}{|l|c|}
\hline ANÁLISIS & RESULTADO \\
\hline Acidez expresada en Ac. citrico & $0,2944 \%$ \\
Azúcares reductores & $4,8900 \%$ \\
pH & $3,94 \%$ \\
Sólidos totales & $15,68 \%$ \\
Sólidos solubles & $15^{\circ}$ Brix \\
\hline
\end{tabular}


5) El tratamiento térmico de $85^{\circ} \mathrm{C}$ por 15 minutos asegura la conservación del néctar.

\section{BIBLIOGRAFÍA}

ALVAREZ, F. Conservación Química: Pulpa de papaya por acción de preservantes. Tesis Ing $^{\circ}$. en Industrias alimentarias, Tingo Maria - Perú. UNAS, 1984.

CALZADA BENZA, José. 143 Frutales Nativos. Distribuidora Estudiante. Primera edición. LimaPerú, 1980.

CALZADA BENZA, José. Métodos estadísticos para la investigación. Lima, 1966.

COLLAZOS, Carlos et al. Composición de los alimentos peruanos. Ministerio de salud. Instituto de nutrición. Reimpresión PEA.1986.

CHEFTEL, Jean-Claude. Introducción a la bioquímica de alimentos. Editorial Acribia. ZaragozaEspaña 1980. Tomo I.

DE FLORIO RAMIREZ, Enrique. Elaboración de jalea, UNJBG. Trabajo de investigación no publicado.

DESROSIER, Norman. Conservación de alimentos. Cía Editorial Continental S.A. México, 1982.

DUCKWORT, R. Frutas y vegetales. Editorial Acribia, España, 1988.

GUEVARA, varios. Procesamiento de fruta, 1989.
6) El uso del CMC tiene que ser acompañado necesariamente por un homogeneizado del producto.

HART, F. L.; FISHER, H. J. Análisis modernos de alimentos. Editorial Acribia, Zarajoza-España, 1984.

HURTADO PASCUAL, Fernando. Ensayo de procesamiento de maracuyá y cocona. Tesis de Ing $^{\circ}$ en Industrias Alimentarias, LA MOLINA UNA, 1968

ITINTEC. Normas técnicas: 203.001, 203.002, 203.011 Lima - Perú, 1984.

LUCK, E. Conservación química de los alimentos. Zaragoza - España. Editorial Acribia, 1971.

MANUAL DE TÉCNICAS AGROPECUARIAS. Conservación de frutas y hortalizas. Editorial Acribia, España, 1976.

MANUAL PARA LA EDUCACIÓN AGROPECUARIA. Elaboración de frutas y hortalizas. Industrias Rurales $N^{\circ} 25$. Editorial Trillas. México 1986.

MINISTERIO DE AGRICULTURA DEL PERÚ. Anuario agropecuario, 1986.

PEARSON, D. Técnicas de laboratorio para el análisis de alimentos. Editorial Acribi, primera reimpresión, Zaragoza - España, 1986.

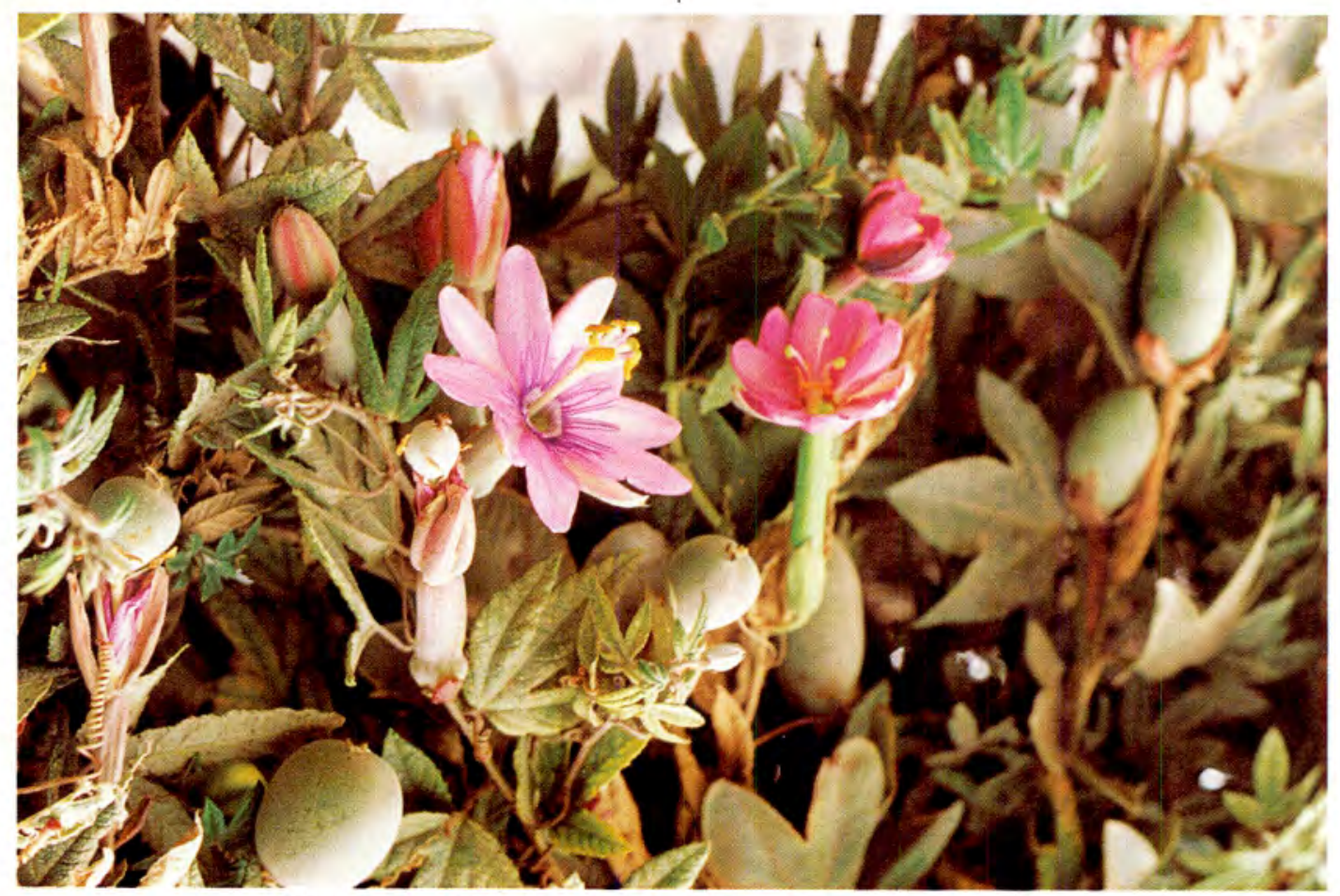

Tumbo serrano. Abunda en el anexo de Ticaco, provincia de Tarata-Tacna. 